\title{
Selective Augmentation of Striatal Functional Connectivity Following NMDA Receptor Antagonism: Implications for Psychosis
}

\author{
Orwa Dandash*,1,2, Ben J Harrison', Ram Adapa ${ }^{3,4}$, Raphael Gaillard ${ }^{5,6,7}$, Francesco Giorlando ${ }^{8}$, \\ Stephen J Wood ${ }^{1,9}$, Paul C Fletcher ${ }^{10}$ and Alex Fornito ${ }^{1,2}$ \\ 'Melbourne Neuropsychiatry Centre, Department of Psychiatry, University of Melbourne, Melbourne, VIC, Australia; ${ }^{2}$ Monash Clinical and Imaging \\ Neuroscience Laboratory, School of Psychology and Psychiatry, Monash University, Clayton, VIC, Australia; ${ }^{3}$ Division of Anaesthesia, University of \\ Cambridge, Cambridge, UK; ${ }^{4}$ Addenbrooke's Hospital, Cambridge, UK; ${ }^{5}$ Université Paris Descartes, Sorbonne Paris Cité, INSERM UMR S894, \\ Paris, France; ${ }^{6}$ Centre Hospitalier Sainte-Anne, Department of Psychiatry, Service Hospitalo-Universitaire, Paris, France; ${ }^{7}$ INSERM, Laboratoire de \\ Physiopathologie des Maladies Psychiatriques, Centre de Psychiatrie et Neurosciences, UMR S894, Paris, France; ${ }^{8}$ Department of Psychiatry, \\ University of Melbourne, Melbourne, VIC, Australia; ${ }^{9}$ School of Psychology, University of Birmingham, Birmingham, UK; ${ }^{10}$ Department of \\ Psychiatry, Brain Mapping Unit and Behavioural and Clinical Neurosciences Institute, School of Clinical Medicine, University of Cambridge, \\ Cambridge, UK
}

The psychotomimetic effect of the $\mathrm{N}$-methyl-D-aspartate receptor (NMDAR) antagonist ketamine is thought to arise from a functional modulation of the brain's fronto-striato-thalamic (FST) circuits. Animal models suggest a pronounced effect on ventral 'limbic' FST systems, although recent work in patients with psychosis and high-risk individuals suggests specific alterations of dorsal 'associative' FST circuits. Here, we used functional magnetic resonance imaging to investigate the effects of a subanesthetic dose of ketamine on measures of functional connectivity as indexed by the temporal coherence of spontaneous neural activity in both dorsal and ventral FST circuits, as well as their symptom correlates. We adopted a placebo-controlled, double-blind, randomized, repeated-measures design in which 19 healthy participants received either an intravenous saline infusion or a racemic mixture of ketamine ( $100 \mathrm{ng} / \mathrm{ml})$ separated by at least I week. Compared with placebo, ketamine increased functional connectivity between the dorsal caudate and both the thalamus and midbrain bilaterally. Ketamine additionally increased functional connectivity of the ventral striatum/nucleus accumbens and ventromedial prefrontal cortex. Both connectivity increases significantly correlated with the psychosis-like and dissociative symptoms under ketamine. Importantly, dorsal caudate connectivity with the ventrolateral thalamus and subthalamic nucleus showed inverse correlation with ketamine-induced symptomatology, pointing to a possible resilience role to disturbances in FST circuits. Although consistent with the role of FST in mediating psychosis, these findings contrast with previous research in clinical samples by suggesting that acute NMDAR antagonism may lead to psychosis-like experiences via a mechanism that is distinct from that implicated in frank psychotic illness. Neuropsychopharmacology (2015) 40, 622-631; doi:I0.1038/npp.2014.210; published online 17 September 20I4

\section{INTRODUCTION}

The $N$-methyl-D-aspartate receptor (NMDAR) hypofunction is thought to play a primary role in the pathophysiology of psychosis (Javitt and Zukin, 1991; Olney and Farber, 1995). Its effects are commonly modeled in healthy volunteers by administration of ketamine, a potent antagonist of NMDARs on cortical GABAergic cells that leads to disinhibited glutamatergic stimulation of non-NMDA (ie,

*Correspondence: O Dandash, Melbourne Neuropsychiatry Centre, Department of Psychiatry, University of Melbourne, Level 3, Alan Gilbert Building, 16I Barry Street, Carlton South, Melbourne, VIC 3053, Australia, Tel: +6I 433 II 7740, Fax: +6I 393480469 , E-mail: orwa.dandash@unimelb.edu.au

Received 8 March 20I4; revised 20 July 20I4; accepted 23 July 20I4; accepted article preview online 21 August 2014 kainate and AMPA) receptors (Moghaddam et al, 1997; Olney and Farber, 1995). These effects have a downstream impact on mesocortical and mesolimbic dopamine pathways (Adams and Moghaddam, 1998; Moghaddam et al, 1997). In addition, ketamine infusion increases blood flow and metabolic activity in the prefrontal cortex, striatum, and thalamus (Holcomb et al, 2001; Vollenweider et al, 1997), the effects that strongly correlate with the emergence of dissociative and psychosis-like symptoms (Driesen et al, 2013; Holcomb et al, 2001; Vollenweider et al, 1997). Disruption of fronto-striato-thalamic (FST) circuitry is thought to play a major role in the pathogenesis of schizophrenia and dissociative phenomena (Pantelis et al, 1992).

FST circuits comprise a set of parallel yet integrated loops serving distinct and complementary functions (Alexander et al, 1986). This functional specificity raises the question of 
whether ketamine exerts its psychiatric effects through selective modulation of activity in one circuit over another. Animal models suggest that NMDAR inhibition leads to schizophrenia-like behavior and increased dopamine in the ventral, the so-called 'limbic' FST circuit, linking the medial prefrontal cortex and the ventral/limbic striatum (ie, nucleus accumbens) (Moghaddam et al, 1997). In contrast, recent human molecular imaging studies have found that both patients with psychotic disorders and those with an 'at-risk mental state' (ARMS) for psychosis show increased dopamine transmission in the dorsal, the so-called 'associative' striatum (Howes et al, 2009; Kegeles et al, 2010), and that these changes correlate with symptom severity and abnormal dorsal prefrontal cortical activation (Fusar-Poli et al, 2011). Such findings suggest that the psychotomimetic effects of ketamine may arise, in part, from its influence on dopamine transmission in dorsal FST circuits. Alternatively, ketamine might induce psychosis-like experiences through an independent mechanism, given evidence that dopamine D2 receptor blockade does not attenuate ketamine-induced psychotic symptoms (Krystal et al, 1999; Lahti et al, 1995).

In light of these possibilities, we sought to characterize the effects of a subanesthetic dose of ketamine on the temporal coherence-ie, functional connectivity-of spontaneous neural activity of dorsal and ventral FST circuits as measured using resting-state functional magnetic resonance imaging (fMRI) in healthy young adults. Resting-state fMRI offers a powerful means to map the functional integrity of large-scale brain networks and their modulation with pharmacological agents (Cole et al, 2012; Driesen et al, 2013). Our own recent work has identified a prominent reduction of functional connectivity in dorsal FST circuits in ARMS subjects, first-episode schizophrenia patients, and their unaffected first-degree relatives (Dandash et al, 2013b; Fornito et al, 2013). As such, if the psychotomimetic effects of ketamine arise through a mechanism similar to that seen in patients with psychosis and ARMS individuals, ketamine should act primarily by reducing functional connectivity in dorsal FST circuitry. A distinct mode of action would be suggested by alternative FST functional connectivity changes.

\section{MATERIALS AND METHODS}

\section{Participants}

A total of 21 participants (10 males; mean age 28.7 years, $\mathrm{SD}=3.2$ years) were recruited via advertisements placed throughout central Cambridge, UK. All participants underwent a screening interview in which they were asked whether they had previously been diagnosed or treated for any mental health problems and whether they had ever taken any psychotropic medications. Participants reporting a personal history of any mental health problems or a history of any treatment were excluded from the study. All participants were right-handed, were free of current of previous psychiatric or neurological disorder or substance abuse problems, and had no history of cardiovascular illness or family history of psychiatric disorder/substance abuse. All participants provided written informed consent in accordance with ethics committee guidelines.

\section{Study Design}

Participants were assessed on two occasions, separated by at least 1 week. On one occasion, they received a continuous computer-controlled intravenous infusion of a racemic ketamine solution $(2 \mathrm{mg} / \mathrm{ml})$ until a targeted plasma concentration of $100 \mathrm{ng} / \mathrm{ml}$ was reached. This concentration was sustained throughout the protocol. A saline infusion was administered on the other occasion. Infusion order was randomly counterbalanced across participants. The infusion was performed and monitored by a trained anesthetist (RA) who was unblinded for safety reasons, but who otherwise had minimal contact with participants. At all other times, participants were supervised by investigators blinded to the infusion protocol (AF and RG). The participants remained blinded until both assessments were completed. All MRI and assessment procedures were identical across assessment occasions.

\section{Infusion Protocol}

Bilateral intravenous catheters were inserted into volunteers' forearms, one for infusion, and the other for serial blood sampling. We used a validated and previously implemented (Corlett et al, 2006) three-compartment pharmacokinetic model to achieve a constant plasma concentration of $100 \mathrm{ng} / \mathrm{ml}$ (Domino et al, 1982) using a computerized pump (Graseby 3500, Graseby Medical, UK). The infusion continued for $15 \mathrm{~min}$ to allow stabilization of plasma levels. Blood samples were drawn before and after the resting $\mathrm{fMRI}$ scan and then placed on ice. Plasma was obtained by centrifugation and stored at $-70^{\circ} \mathrm{C}$. Plasma ketamine concentrations were measured by gas chromatography-mass spectrometry.

\section{Clinical Measures}

Once the infusion had stabilized and before MRI, participants underwent a clinical rating of positive psychotic symptoms as assessed by the Rating Scale for Psychotic Symptoms (RSPS) (Chouinard and Miller, 1999) and the Brief Psychiatric Rating Scale (BPRS) expanded version 4 (Ventura et al, 1993). Seven key items on the BPRS representing positive symptoms of the psychosis prodrome (somatic concerns, anxiety, depression, elevated mood, grandiosity, hallucination, and unusual thought content) were selected. Dissociative symptoms were assessed by the Clinician Administered Dissociative States Scale (CADSS) (Bremner et al, 1998). In addition, participants completed assessments of psychosis proneness (Chapman magical ideation (Eckblad and Chapman, 1983) and perceptual aberration scales (Chapman et al, 1978) and the Peters delusion inventory (Peters et al, 1999)) before the first scan (see Supplementary Materials and Methods, Supplementary Table S1, and Supplementary Figure S2 for further information on scoring properties as well as distribution and descriptive statistics of the psychosis-proneness scales).

\section{MRI Acquisition}

Scanning was performed using a $3.0 \mathrm{~T}$ MRI scanner (Siemens Magnetom, Trio Tim, Erlangen, Germany) 
equipped with a 12-channel array coil located at the Wolfson Brain Imaging Centre, Addenbrooke's Hospital, Cambridge, UK. T2*-weighted echo-planar images were acquired under eyes-closed resting-state conditions. Participants were instructed to close their eyes and let the minds wander without going to sleep. Subsequent participant debriefing ensured that no participants fell asleep during the scan. Imaging parameters were: $3 \times 3 \times 3.75 \mathrm{~mm}$ voxel size, with a time-to-repetition (TR) of $2000 \mathrm{~ms}$, time-to-echo (TE) of $30 \mathrm{~ms}$, flip angle of $78^{\circ}$ in $64 \times 64$ matrix size, and $240 \mathrm{~mm}$ field of view (FOV). A total of 300 volumes comprising 32 slices each were obtained. In addition, highresolution anatomical $\mathrm{T} 1$ images were acquired using a three-dimensional magnetic-prepared rapid gradient echo (MPPRAGE) sequence. In all, 176 contiguous sagittal slices of $1.0 \mathrm{~mm}$ thickness using a TR of $2300 \mathrm{~ms}$, TE of $2.98 \mathrm{~ms}$, flip angle of $9^{\circ}$, and a FOV of $256 \mathrm{~mm}$ in $240 \times 256$ matrix were acquired with a voxel size of $1.0 \mathrm{~mm}^{3}$.

\section{Preprocessing}

An established procedure was used to characterize corticostriatal functional connectivity in relation to six seed regions located in ventral and dorsal areas of the caudate nucleus and putamen per hemisphere (Dandash et al, 2013b; Di Martino et al, 2008; Fornito et al, 2013). Seeds were defined in both hemispheres as $3.5 \mathrm{~mm}$ radial spheres at the following stereotaxic coordinates: dorsal caudate $(x= \pm 13, y=15, z=9)$; ventral striatum/nucleus accumbens $(x= \pm 9, y=9, z=-8)$; dorsal-caudal putamen $(x= \pm 28, y=1, z=3)$, and ventral-rostral putamen $(x= \pm 20, y=12, \quad z=-3)$. Image preprocessing was performed using statistical parametric mapping software (SPM8) and included motion correction and coregistration of functional images with subjects' anatomical scans that were concurrently normalized to SPM-T1 template. The resulting transformation matrix was applied to the functional data to achieve accurate spatial normalization across individuals. The anatomical scans were also segmented using a unified normalization and segmentation approach. Segmented white matter and CSF images were thresholded at $50 \%$ tissue probability and binarized to create nuisance variable masks, together with a binary mask of the global brain volume (summed from the gray matter, white matter, and CSF segments). Although it has been suggested that the removal of the global signal can possibly induce negative or anti-correlated relationships between brain regions (Murphy et al, 2009), a large body of competing evidence suggests that the removal of the global signal significantly improves the specificity of resting-state fMRI results (see Van Dijk et al, 2010 for a detailed analysis and an extensive list of supporting evidence). In addition, it is highly unlikely that the between-condition comparisons are confounded by this preprocessing step, given that the removal of the global signal was identically performed across both conditions, ketamine and placebo.

Functional images were smoothed using a Gaussian filter (full width at half maximum, $8 \mathrm{~mm}$ ). All image sequences were routinely inspected for potential normalization artifacts. Mean time series were then extracted from each seed region and each nuisance mask for each participant. Two participants were excluded for excessive head motion
( $>2 \mathrm{~mm}$ translation and $>2^{\circ}$ rotation), resulting in a final sample of 19 subjects.

\section{First-Level, Within-Subject Analysis}

For each participant, functional connectivity maps were estimated using whole-brain general linear models as implemented in SPM8. Before model estimation, each of the three nuisance covariates (white matter, cerebrospinal fluid, and global mean signals) and six head motion parameters (three translation and three rotation) were orthogonalized with respect to each seed's time series, resulting in a model comprising six 'noise-cleaned' regions and nine nuisance variables. Time courses extracted from each striatal subregion were entered into a single general linear model that included all striatal seeds together with the nine nuisance variables and used as a variable of interest in a whole-brain regression analysis. Data were high-pass filtered ( $\sim 0.008 \mathrm{~Hz}$ cutoff) and contrast images were generated for each participant by estimating the regression coefficient between all brain voxels and each region's time series separately for left and right hemisphere seeds. The results were largely replicated when employing a more comprehensive first-level motion correction procedure as recently recommended (Satterthwaite et al, 2013; see Supplementary Figure S1).

\section{Second-Level, Between-Condition Analysis}

For each striatal region, participant-specific contrast images were included in a random-effects $2 \times 2$ factorial design (Condition (placebo, ketamine) by Hemisphere (left, right)). Within-condition statistical maps were thresholded at a false discovery rate (FDR) of $P<0.05$ for the whole brain volume (Figure 1). Between-condition effects (main effects of condition and condition $\times$ hemisphere interactions) were mapped by implicitly masking the $t$-contrasts (one tailed) with a global conjunction of the within-subject striatal seed main effect across ketamine and placebo for the combined left and right hemispheres. Nuisance covariates included four additional summary measures of head motion unaccounted for by the first-level correction procedure (Van Dijk et al, 2012). These covariates included: (1) the number of significant micromovements (instances of $>0.10 \mathrm{~mm}$ relative displacement between adjacent volumes), (2) mean head displacement, (3) maximum head displacement, and (4) mean head rotation. Consistent with our past work (Dandash et al, 2013a; Fornito et al, 2013), between-condition statistical maps were thresholded using a $P<0.05$ family-wise error (FWE) cluster corrected threshold determined by a permutation test based on the AlphaSim algorithm (1000 permutations with a clusterforming threshold of $P<0.01$ uncorrected), as implemented in the REST toolbox (Song et al, 2011). A more stringent cluster-forming threshold of $P<0.001$ uncorrected was additionally used to test the robustness of the findings (see Results).

\section{Brain-Behavior Analysis}

The difference between symptom scores obtained under placebo and ketamine (ketamine-placebo) was computed to 


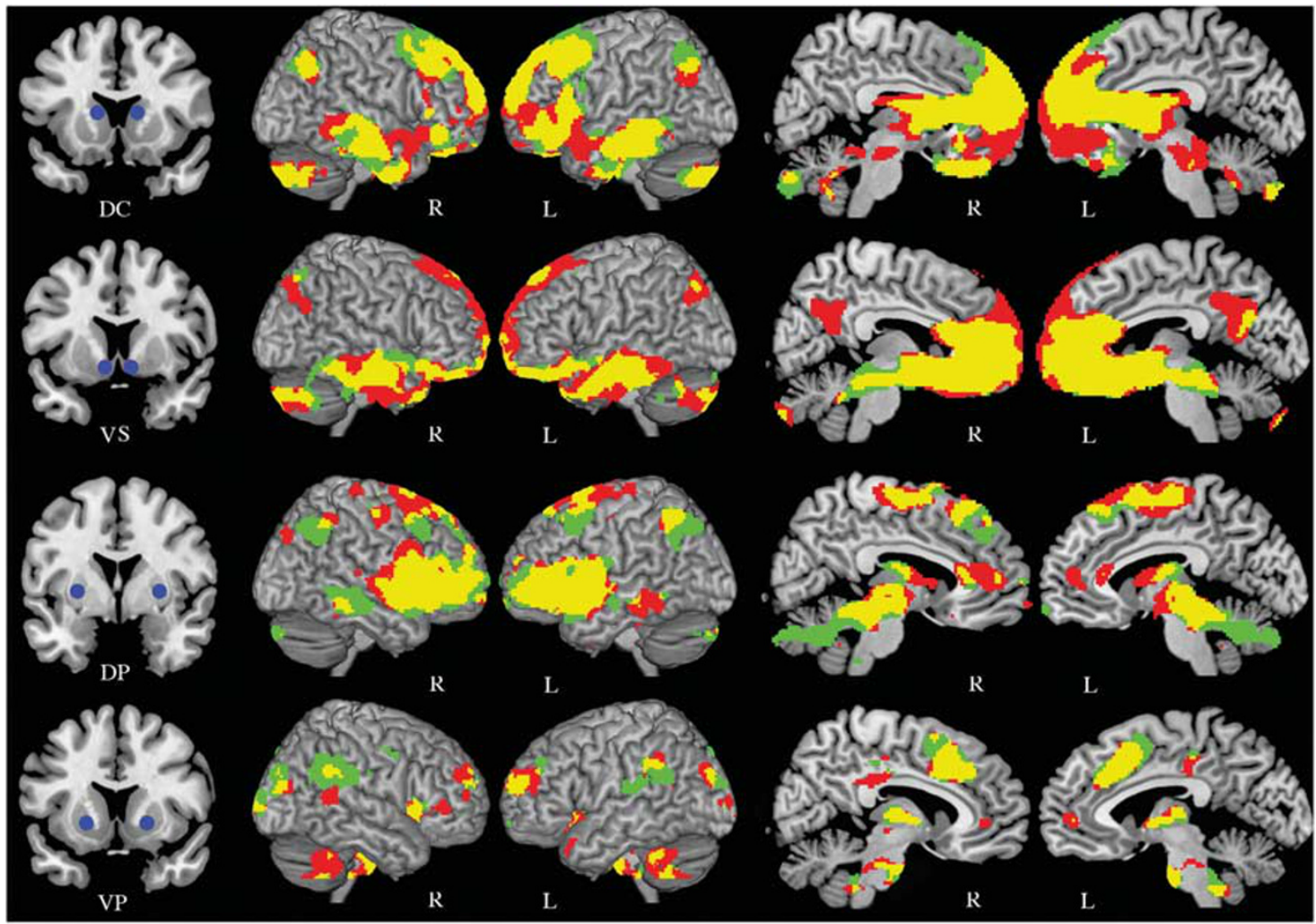

Figure I Significant within-group functional connectivity maps of the dorsal caudate (DC), ventral striatum/nucleus accumbens (VS), dorsal putamen (DP), and ventral putamen (VP) seeds (in blue). Green indicates connectivity under placebo, red indicates connectivity under ketamine infusion, and yellow indicates areas of overlap. R, right hemisphere; L, left hemisphere. Sagittal slices are displayed at $\times= \pm 6$. Because of the large magnitude of seed main effects, these maps were corrected using voxel-wise threshold of $P<0.05$ (FDR corrected).

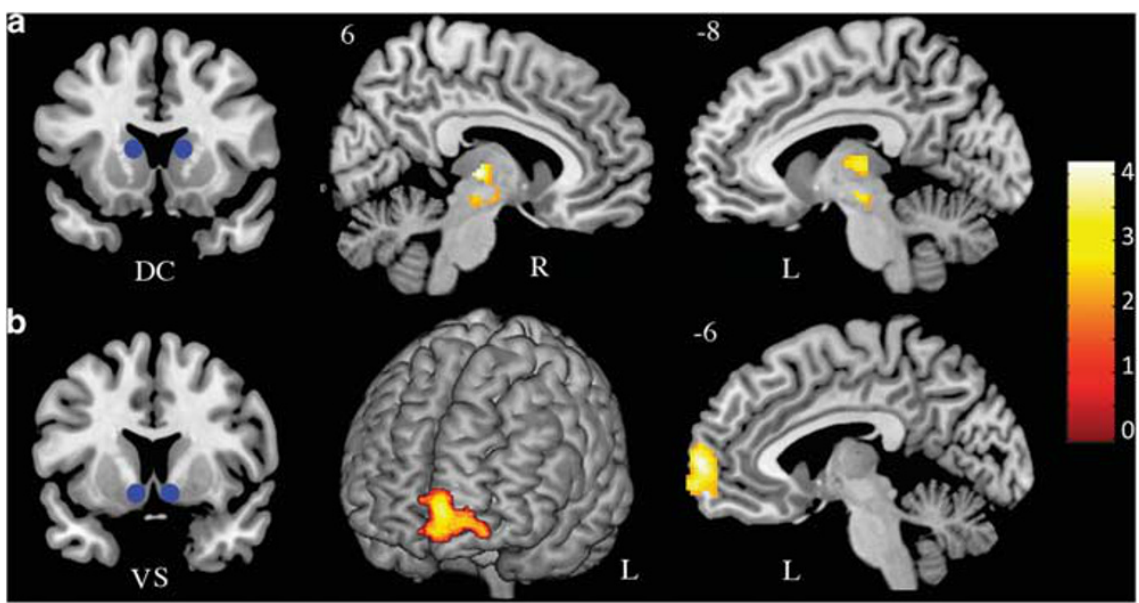

Figure 2 Z-score statistical maps of significant between-condition differences in functional connectivity of the (a) dorsal caudate (DC) and (b) ventral striatum (VS). R, right hemisphere; L, left hemisphere. Coordinates correspond to MNI space. Results are displayed at $P<0.05$ (FWE cluster corrected).

obtain change scores for each scale, denoted by $\triangle$ BPRS, $\triangle \mathrm{RSPS}$, and $\triangle \mathrm{CADSS}$. These change scores were correlated, on a voxel-wise basis, with subject-specific parameter estimates for the effect of ketamine ( $v s$ placebo) on striatal functional connectivity, masked by regions showing a significant ketamine effect on functional connectivity (Figure 2 and Table 2). The four aforementioned summary measures of head motion were included as nuisance variables in these analyses. All results were displayed at a FWE cluster corrected level of $P<0.05$, with appropriate correction for the search volume employed. Secondary analyses tested for associations between functional connectivity changes and trait-like measures of psychosis proneness (ie, the Chapman and Peters scales). 
Table I Symptom Scores of Healthy Participants Before and After the Administration of Ketamine

\begin{tabular}{lccc}
\hline Psychotic and dissociative symptoms measures $(\boldsymbol{n}=\mathbf{1 9})$ & Placebo mean (SD) & Ketamine mean (SD) & Wilcoxon signed-ranks Z, $\boldsymbol{P}$ \\
\hline Brief Psychotic Rating Scale (BPRS) & $7.7(2.3)$ & $9.7(3.7)$ & $-2.53, P=0.011$ \\
Rating Scale for Psychotic Symptoms (RSPS) & $2.3(4.3)$ & $20.2(16.8)$ & $-3.55, P<0.001$ \\
Clinician Administered Dissociative States Scale (CADSS) & $0.5(1.2)$ & - & - \\
Psychosis-proneness measures ( $\mathbf{n}=\mathbf{I 6})^{\mathbf{a}}$ & Baseline mean (SD) & - & - \\
Chapman Magical Ideation scale & $3.5(3.6)$ & - & - \\
Chapman Perceptual Aberration scale & $4.1(6.7)$ & - \\
Peters Delusion Inventory & $36.8(31.1)$ & - \\
\hline
\end{tabular}

avalues for three subjects were not available.

Table 2 Brain Regions Demonstrating Significant Between-Condition (Ketamine Greater Than Placebo) Differences in Functional Connectivity and Association with Symptom Change Scores (Ketamine-Placebo)

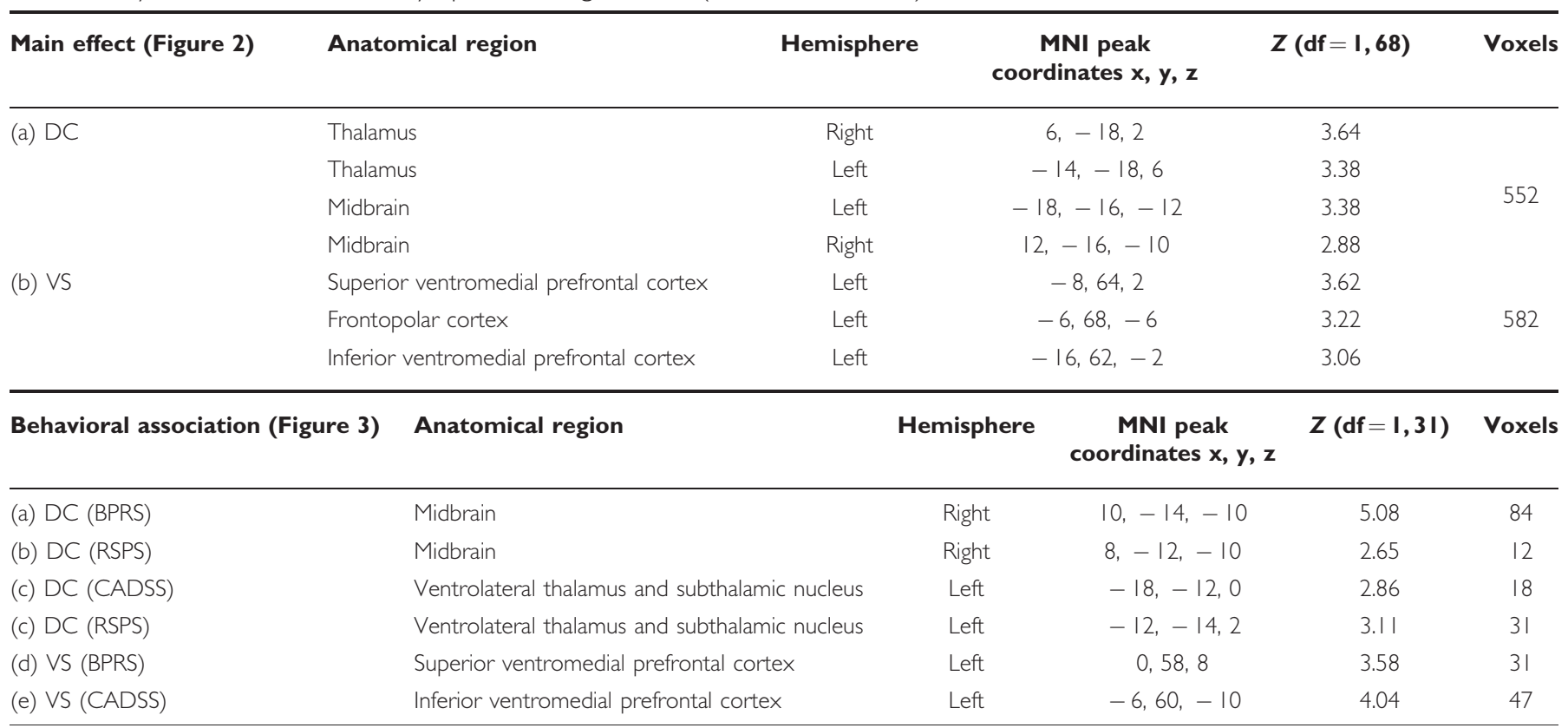

Abbreviation: DC, dorsal caudate; VS, ventral striatum.

Results are thresholded at $P<0.05$ (FWE) cluster corrected.

\section{RESULTS}

\section{Plasma Concentrations and Symptom Ratings}

The mean plasma concentration of ketamine before and after scanning was $68.6 \pm 43.6 \mathrm{ng} / \mathrm{ml}$. Ketamine caused a significant increase in psychosis-like and dissociative symptoms as indexed by the RSPS, BPRS, and the CADSS, respectively (Table 1). Endorsed RSPS items pertained mainly to somatosensory perturbations and loss of attention focus; BPRS items included grandiosity, elevated mood, anxiety, delusional thoughts, and hallucinations; and CADSS items pertained to external and reality perception. These symptoms concur with previous reports (Krystal et al, 1994). Ketamine-induced symptomatology was not accompanied by changes in head motion as found by a formal between-condition comparison.

\section{Functional Connectivity Analyses}

Collapsed across placebo and ketamine conditions, the four seed-specific striatal functional connectivity maps closely recapitulated previous studies (Figure 1; Di Martino et al, 2008; Harrison et al, 2009). Compared with placebo, ketamine augmented functional connectivity of the dorsal caudate and ventral striatum/nucleus accumbens seeds only (Figure 2 and Table 2). Specifically, functional connectivity was increased between the dorsal caudate and regions in the thalamus and midbrain bilaterally (Figure 2a). Connectivity was also increased between the ventral striatum and left anterior and ventromedial prefrontal cortex (Figure $2 b$ ). All effects except for the ketamine-induced increase of dorsal caudate-midbrain connectivity were replicated using a cluster-forming threshold of $P=0.001 \quad(P<0.05$, FWE cluster corrected). There were no significant effects of 


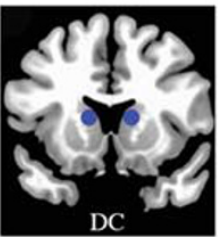

a

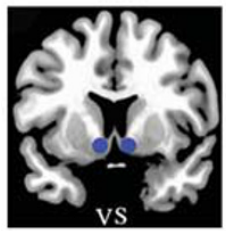

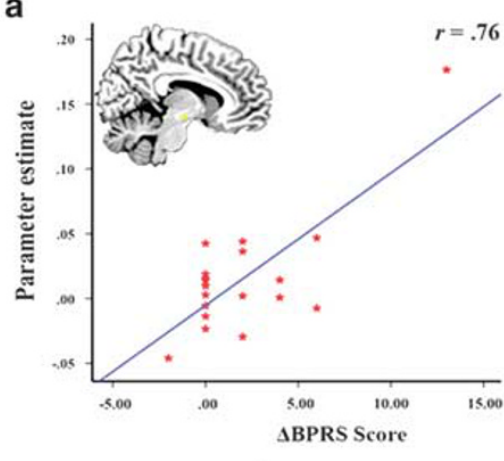

b

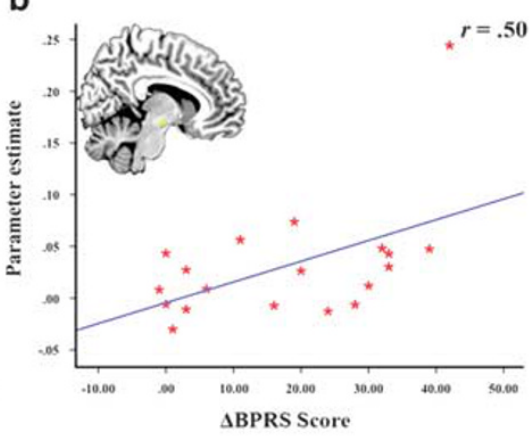

C

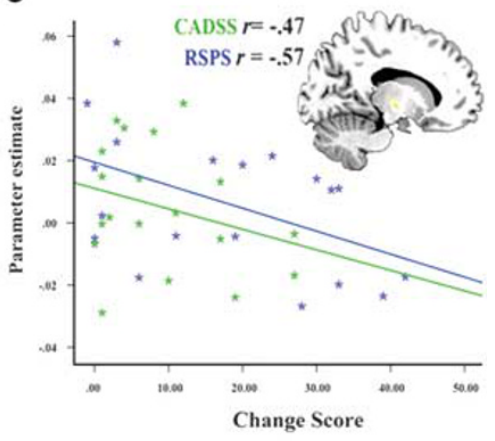

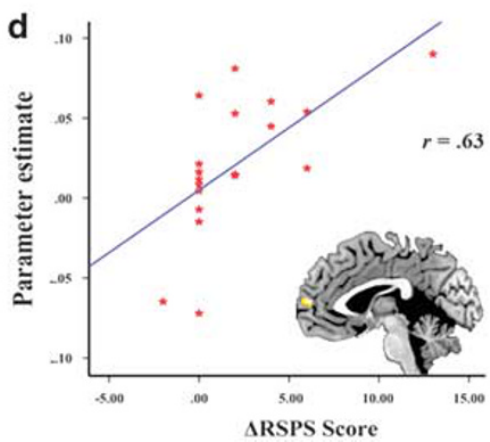

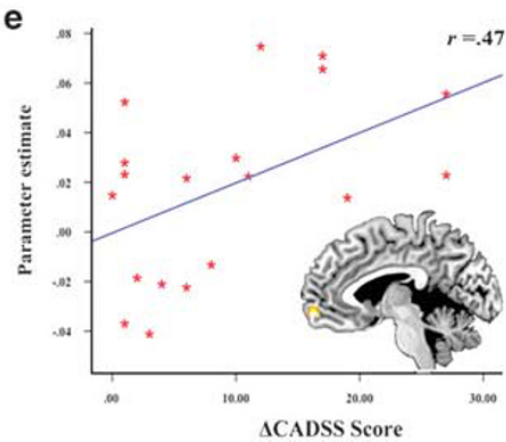

Figure 3 Z-scores statistical maps of significant brain-behavior association between estimates of functional connectivity of striatal seeds and positive psychotic and dissociative symptoms caused by ketamine infusion, displayed at $P<0.05$ (FWE cluster corrected). For visualizing purposes with the midbrain ( $a$ and b), ventral thalamus/subthalamic nucleus (c) and medial prefrontal cortex ( $d$ and e), parameter estimates were extracted from peak voxels in the shown brain maps and correlated with symptom change scores. Results remained significant after the exclusion of outliers.

ketamine on putamen functional connectivity, no significant condition-by-hemisphere interactions, and no significant functional connectivity decreases observed under ketamine relative to placebo.

\section{Brain-Behavior Associations}

A stronger ketamine-related increase of functional connectivity between the dorsal caudate and right midbrain was correlated with higher $\triangle$ BPRS scores (Figure 3a). Conversely, a stronger effect of ketamine on functional connectivity between dorsal caudate and left ventrolateral thalamus, as well as between dorsal caudate and subthalamic nucleus, was associated with lower $\triangle$ RSPS and lower $\triangle$ CADSS scores (Figure $3 \mathrm{~b}$ ), suggesting a possible protective role in combating the psychotogenic effect of ketamine. In the ventral system, higher ketamine-induced functional connectivity between ventral striatum and left ventromedial prefrontal cortex was associated with higher $\triangle \mathrm{BPRS}$ scores (Figure 3c), whereas higher $\triangle$ CADSS scores were associated with a stronger ketamine effect on functional connectivity between the ventral striatum and an adjacent ventromedial prefrontal region (Figure 3d). All results were FWE cluster corrected $(P<0.05)$ for multiple comparisons. Theoretical and empirical models suggest a competitive or antagonistic relationship between dorsal and ventral frontostriatal function (O'Doherty et al, 2004). In support of this view, an exploratory analysis of the association between participant-specific parameter estimates of ketamineinduced functional connectivity changes in dorsal (dorsal caudate-subthalamic nucleus connectivity) and ventral (ventral striatal-frontopolar cortex connectivity) systems found a significant negative correlation $(r=-0.5$, $P<0.029)$. In other words, ketamine-related enhancement of connectivity in one circuit reduced connectivity in the other.

No significant associations were observed between measures of psychosis proneness (ie, the Chapman and Peters scales) and ketamine-induced functional connectivity changes. However, exploratory analyses revealed a significant association between the Chapman magical ideation and RSPS difference scores $(r=0.51, P<0.045)$. A trend was also observed between Chapman perceptual abnormality and RSPS scores $(r=0.48, P<0.058)$, suggesting that individuals who had higher psychotic traits experienced more severe psychotic-like symptoms under ketamine.

\section{DISCUSSION}

Ketamine increased functional connectivity between specific components of both dorsal and ventral FST circuits and these increases correlated with the emergence of psychosislike and dissociative phenomena assessed with the BPRS, RSPS, and the CADSS, respectively. These drug-induced functional connectivity changes contrast with the alterations found in patients with first-episode psychosis, their unaffected relatives, and ARMS individuals (Dandash et al, 2013b; Fornito et al, 2013) who show reduced functional connectivity in dorsal FST systems and less consistent evidence for ventral circuit alterations (Dandash et al, 2013b; Fornito et al, 2013). Specifically, two primary differences were noted. First, ketamine led to a general increase, rather than decrease, in both dorsal and ventral 
circuit functional connectivity. Second, the dorsal circuit increases were primarily subcortical, affecting functional coupling of the dorsal caudate with midbrain and thalamus (Figure 2a), rather than the frontostriatal changes seen in clinical populations. These findings imply that the psychosis-like experiences elicited by an acute dose of ketamine arise from FST alterations that are distinct from those associated with the primary pathophysiological disturbance in psychosis.

The general increase in FST functional connectivity is consistent with past reports that ketamine augments regional resting cerebral blood flow, metabolic and electrophysiological activity, and resting-state functional connectivity in these and other regions (Driesen et al, 2013; Holcomb et al, 2001; Vollenweider et al, 1997). This effect is thought to arise from excitation-inhibition imbalance (Anticevic et al, 2012) caused by ketamine's blockade of NMDARs on inhibitory GABAergic interneurons that leads to the disinhibition of glutamate release, resulting in excess glutamatergic binding at AMPA and kainate receptors (Moghaddam et al, 1997). The striatum receives afferent glutamatergic input from the prefrontal cortex and sends efferents to the thalamus via the pallidum and midbrain. The thalamus projects back to the originating prefrontal area completing a FST loop (Alexander et al, 1986). Influential models of psychosis posit that increased flow of sensory information from the striatum to the thalamus results in a failure of the thalamus to 'filter-out' irrelevant stimuli before they reach the cortex, thus predisposing individuals to psychotic symptoms (Carlsson, 1988). The associations we found between the emergence of psychosislike symptoms and ketamine-induced increases of functional connectivity (Figure 3) support this view, under the assumption that increased functional connectivity reflects increased AMPA/kainate-mediated feed-forward frontostriatal and striatothalamic signaling. However, this result is at odds with the lack of subcortical functional connectivity increases observed in first-episode patients and ARMS individuals (Dandash et al, 2013b; Fornito et al, 2013).

Dysregulation of dopamine likely plays a central role in the risk-related FST changes observed in clinical samples, given robust evidence for elevated dopaminergic function, particularly in dorsal striatal regions, in both patients (Kegeles et al, 2010) and high-risk individuals (Howes et al, 2009). Either too much or too little dopamine in FST circuits is expected to reduce neural signal-to-noise ratio (Cools and D'Esposito, 2011) and thus lower functional connectivity (Fornito et al, 2013). NMDAR hypofunction has been implicated as a candidate upstream cause of dopaminergic abnormalities associated with psychosis onset (Carlsson and Carlsson, 1990) and some human studies suggest that ketamine augments dopamine release in a manner that parallels observations in clinical sample (Kegeles et al, 2000), while also affecting activation of frontostriatal systems (Corlett et al, 2006). However, other studies have failed to demonstrate that ketamine increases striatal dopamine transmission, even with very high doses (Aalto et al, 2002), that ketamine has a limited effect on D2 receptor availability (Kegeles et al, 2002), and that treatment with Haloperidol has no impact on ketamine-induced psychotic symptoms (Krystal et al, 1999; Lahti et al, 1995). These findings suggest a mode of action for ketamine that diverges from the pathophysiological alterations seen in psychosis patients.

Although our findings support a distinct mode of action for ketamine, two considerations merit discussion. The first pertains to dosage. Participants in our study received a lower dose of ketamine than is traditionally used (Aalto et al, 2002; Kegeles et al, 2002; Krystal et al, 1994) to maximize in-scanner tolerance. Although the dose was sufficient to elicit an increase in psychosis-like and dissociative symptoms, their intensity and quality may have been more characteristic of an ARMS than frank psychotic episode. Nonetheless, dorsal FST functional connectivity reductions have still been observed in ARMS individuals (Dandash et al, 2013b) and symptom-free genetic high-risk volunteers (Fornito et al, 2013), suggesting such changes are present at the earliest signs of illness. Moreover, functional connectivity reductions are by far the most common finding in patients with psychosis across different illness stages and neural systems (Pettersson-Yeo et al, 2011), contrasting the generic functional connectivity increases observed in this study and elsewhere, even when using much higher doses $(\sim 162 \mathrm{ng} / \mathrm{ml}$ )(Driesen et al, 2013). These results, combined with other evidence that the dose we used is sufficient to effectively model other psychosis-related brain changes in healthy volunteers (Corlett et al, 2006), support the conclusion that not all of the neural effects of ketamine adequately model the brain changes seen in clinical samples, even when they correlate with drug-induced increases in symptomatology (Pomarol-Clotet et al, 2006). Thus, ketamine may provide a better model of some riskrelated brain changes than others.

A second consideration is regarding the chronicity of exposure. It has been argued that acute NMDAR hypofunction may model neural changes associated with cognitive disturbances that pre-date psychosis onset (eg, akin to an ARMS) (Corlett et al, 2007), whereas longer-term deficits may be more characteristic of schizophrenia (Freeman et al, 2009). In this regard, early hyperconnectivity of FST circuits may evolve into subsequent hypoconnectivity as psychosis takes hold. This view is supported by reports that striatal glutamate is elevated in early stages of schizophrenia but reduces with antipsychotic treatment (de la FuenteSandoval et al, 2013), that administration of ketamine is associated with reduced functional connectivity in some brain circuits after $24 \mathrm{~h}$ (Scheidegger et al, 2012), and that chronic but not acute use of ketamine leads to delusion formation (Freeman et al, 2009) that persists after abstinence (Morgan et al, 2010). However, the fact that dorsal FST hypoconnectivity is observed in ARMS individuals (Dandash et al, 2013b) and symptom-free first-degree relatives of patients populations (Fornito et al, 2013) that putatively represent the earliest illness stages suggests that acute ketamine infusion provides an inadequate model of risk-related FST dysconnectivity. Whether chronic exposure better models these changes is an open question.

Interestingly, although ketamine-related increases of functional connectivity were generally associated with more severe psychosis-like and dissociative symptoms, increased connectivity between dorsal caudate and regions of the thalamus and subthalamic nucleus was associated with lower symptom scores (Figure $3 \mathrm{~b}$ ). Others have found that ketamine-induced negative symptoms inversely correlate 
with functional connectivity changes of the ventrolateral thalamus with the rest of the brain (Driesen et al, 2013). The thalamus and subthalamic nucleus are thought to exert modulatory feedback over striatal output within corticostriato-thalamic circuits (Parent and Hazrati, 1995). Although the modulatory role of the ventrolateral thalamus on dorsal striatum is less clear, glutamatergic cells in the thalamus and subthalamic nucleus (STN) are proposed to initiate a cascade of events resulting in activation of GABAergic cells in the pallidum, thus dampening outflow from the striatum (Carlsson, 1988; Carlsson and Carlsson, 1990). This feedback is believed to prevent overflow of sensory information, and its failure has been implicated in vulnerability to psychotic experiences (Carlsson and Carlsson, 1990). Accordingly, our findings indicate that individuals who engaged this system to a greater extent experienced fewer psychosis-like and dissociative symptoms under ketamine. Upregulating functional connectivity in this circuit may represent a viable treatment target to combat the deleterious effects of dysfunction elsewhere in the system. This view is consistent with our finding that a stronger ketamine-related increase of dorsal caudate-STN connectivity was associated with a reduced ketamineinduced increase of ventral striatum-frontopolar cortex connectivity, a result that further points to some degree of functional antagonism between these two circuits in mediating the psychotogenic effects of ketamine (O'Doherty et al, 2004).

Although we have focused on the known influence of ketamine on ionotropic glutamate receptors and dopaminergic signaling, the drug can affect other modulatory systems (Kapur and Seeman, 2002). However, the average dose used herein provides $<0.1 \mu \mathrm{M}$ available for binding (assuming plasma binding value of $47 \%$ (Dayton et al, 1983) and a molecular weight of 274.19 (ketamine hydrochloride)). At this concentration, ketamine demonstrates no binding potential for most other receptors (eg, $\mu$-opioid, sigma, dopamine, or 5-HT; Kapur and Seeman, 2002; Smith et al, 1987).

In summary, our findings suggest that ketamine affects functional connectivity of both dorsal and ventral FST circuits and that these changes correlate with the severity of psychosis-like and dissociative symptoms induced by the drug. They also point to a potential role for the ventrolateral thalamus and subthalamic nucleus in protecting against disturbances that lead to psychotic experience. Although consistent with the role of FST in mediating psychosis, these effects appear somewhat distinct from the FST changes observed in first-episode patients, unaffected relatives, and ARMS individuals, suggesting that acute ketamine infusion does not completely model the disturbances of FST functional connectivity implicated in risk for psychosis. Investigating the impact of NMDAR agonists as well as glutamate antagonists on the functional connectivity of the ventrolateral thalamus and subthalamic nucleus is important to further delineate their protective role.

\section{FUNDING AND DISCLOSURE}

RG received compensations from Astra Zeneca, Bristol Myer Squib, Janssen, Lundbeck, Otsuka, Roche, Sanofi,
Servier, and Takeda, and a grant from the Centre de Psychiatrie et Neurosciences, Sainte Anne, Paris. FG received scholarships from the University of Melbourne (Melbourne Research Scholarship) and the Australasian Society for Bipolar \& Depressive Disorders (ASBDD/ AstraZeneca Scholarship). He also received a Pfizer Neuroscience Research Grant and a RANZCP (Royal Australian and New Zealand College of Psychiatrists) Young Investigator Grant. PCF received consultancy fees from GlaxoSmithKline. The other authors declare no conflict of interest.

\section{ACKNOWLEDGEMENTS}

The study was funded by the Bernard Wolfe Health Neuroscience Fund and the Wellcome Trust. OD was supported by the Australian Postgraduate Award (APA, 2010). BJH was supported by a National Health and Medical Research Council of Australia (NHMRC) Clinical Career Development Fellowship (ID 628509). RA was supported by the Wellcome Trust Research Training Fellowship (grant no. 083660/Z/07/Z), Raymond and Beverly Sackler Studentship, and the Cambridge Commonwealth Trust. AF was supported by the NHMRC (ID 1050504) and a Monash University Larkins Fellowship.

\section{REFERENCES}

Aalto S, Hirvonen J, Kajander J, Scheinin H, Nagren K, Vilkman H et al (2002). Ketamine does not decrease striatal dopamine $\mathrm{D}(2)$ receptor binding in man. Psychopharmacology 164: 401-406.

Adams B, Moghaddam B (1998). Corticolimbic dopamine neurotransmission is temporally dissociated from the cognitive and locomotor effects of phencyclidine. J Neurosci 18: 5545-5554.

Alexander GE, DeLong MR, Strick PL (1986). Parallel organization of functionally segregated circuits linking basal ganglia and cortex. Annu Rev Neurosci 9: 357-381.

Anticevic A, Gancsos M, Murray JD, Repovs G, Driesen NR, Ennis DJ et al (2012). NMDA receptor function in large-scale anticorrelated neural systems with implications for cognition and schizophrenia. Proc Natl Acad Sci USA 109: 16720-16725.

Bremner JD, Krystal JH, Putnam FW, Southwick SM, Marmar C, Charney DS et al (1998). Measurement of dissociative states with the Clinician-Administered Dissociative States Scale (CADSS). J Trauma Stress 11: 125-136.

Carlsson A (1988). The current status of the dopamine hypothesis of schizophrenia. Neuropsychopharmacology 1: 179-186.

Carlsson M, Carlsson A (1990). Interactions between glutamatergic and monoaminergic systems within the basal ganglia-implications for schizophrenia and Parkinson's disease. Trends Neurosci 13: $272-276$

Chapman LJ, Chapman JP, Raulin ML (1978). Body-image aberration in schizophrenia. J Abnorm Psychol 87: 399-407.

Chouinard G, Miller R (1999). A rating scale for psychotic symptoms (RSPS) part I: theoretical principles and subscale 1: perception symptoms (illusions and hallucinations). Schizophr Res 38: 101-122.

Cole DM, Oei NY, Soeter RP, Both S, van Gerven JM, Rombouts SA et al (2012). Dopamine-dependent architecture of corticosubcortical network connectivity. Cereb Cortex.

Cools R, D’Esposito M (2011). Inverted-U-shaped dopamine actions on human working memory and cognitive control. Biol Psychiatry 69: E113-E125. 
Corlett PR, Honey GD, Aitken MR, Dickinson A, Shanks DR, Absalom AR et al (2006). Frontal responses during learning predict vulnerability to the psychotogenic effects of ketamine: linking cognition, brain activity, and psychosis. Arch Gen Psychiatry 63: 611-621.

Corlett PR, Honey GD, Fletcher PC (2007). From prediction error to psychosis: ketamine as a pharmacological model of delusions. J Psychopharmacol 21: 238-252.

Dandash O, Fornito A, Lee J, Keefe RS, Chee MW, Adcock RA et al (2013a). Altered striatal functional connectivity in subjects with an at-risk mental state for psychosis. Schizophr Bull 40: 904-913.

Dandash O, Fornito A, Lee J, Keefe RSE, Chee MWL, Adcock RA et al (2013b). Altered striatal functional connectivity in subjects with an at-risk mental state for psychosis. Schizophr Bull Online Pub doi:10.1093/schbu/sbt093.

Dayton PG, Stiller RL, Cook DR, Perel JM (1983). The binding of ketamine to plasma-proteins: emphasis on human-plasma. Eur J Clin Pharmacol 24: 825-831.

de la Fuente-Sandoval C, Leon-Ortiz P, Azcarraga M, Stephano S, Favila R, Diaz-Galvis L et al (2013). Glutamate levels in the associative striatum before and after 4 weeks of antipsychotic treatment in first-episode psychosis: a longitudinal proton magnetic resonance spectroscopy study. JAMA Psychiatry 70: 1057-1066.

Di Martino A, Scheres A, Margulies DS, Kelly AM, Uddin LQ, Shehzad $\mathrm{Z}$ et al (2008). Functional connectivity of human striatum: a resting state FMRI study. Cereb Cortex 18: 2735-2747.

Domino SE, Domino LE, Domino EF (1982). Comparison of two and three compartment models of phencyclidine in man. Subst Alcohol Actions Misuse 3: 205-211.

Driesen NR, McCarthy G, Bhagwagar Z, Bloch M, Calhoun V, D'Souza DC et al (2013). Relationship of resting brain hyperconnectivity and schizophrenia-like symptoms produced by the NMDA receptor antagonist ketamine in humans. Mol Psychiatry 18: 1199-1204.

Eckblad M, Chapman LJ (1983). Magical ideation as an indicator of schizotypy. J Consult Clin Psych 51: 215-225.

Fornito A, Harrison BJ, Goodby E, Dean A, Ooi C, Nathan PJ et al (2013). Functional dysconnectivity of corticostriatal circuitry as a risk phenotype for psychosis. JAMA Psychiatry 70: 1143-1151.

Freeman TP, Morgan CJ, Klaassen E, Das RK, Stefanovic A, Brandner B et al (2009). Superstitious conditioning as a model of delusion formation following chronic but not acute ketamine in humans. Psychopharmacology 206: 563-573.

Fusar-Poli P, Howes OD, Allen P, Broome M, Valli I, Asselin MC et al (2011). Abnormal prefrontal activation directly related to pre-synaptic striatal dopamine dysfunction in people at clinical high risk for psychosis. Mol Psychiatry 16: 67-75.

Harrison BJ, Soriano-Mas C, Pujol J, Ortiz H, Lopez-Sola M, Hernandez-Ribas R et al (2009). Altered corticostriatal functional connectivity in obsessive-compulsive disorder. Arch Gen Psychiatry 66: 1189-1200.

Holcomb HH, Lahti AC, Medoff DR, Weiler M, Tamminga CA (2001). Sequential regional cerebral blood flow brain scans using PET with $\mathrm{H} 2(15) \mathrm{O}$ demonstrate ketamine actions in CNS dynamically. Neuropsychopharmacology 25: 165-172.

Howes OD, Montgomery AJ, Asselin MC, Murray RM, Valli I, Tabraham $\mathrm{P}$ et al (2009). Elevated striatal dopamine function linked to prodromal signs of schizophrenia. Arch Gen Psychiatry 66: $13-20$.

Javitt DC, Zukin SR (1991). Recent advances in the phencyclidine model of schizophrenia. Am J Psychiatry 148: 1301-1308.

Kapur S, Seeman P (2002). NMDA receptor antagonists ketamine and PCP have direct effects on the dopamine D-2 and serotonin 5-HT2 receptors-implications for models of schizophrenia. Mol Psychiatry 7: 837-844.

Kegeles LS, Abi-Dargham A, Frankle WG, Gil R, Cooper TB, Slifstein $\mathrm{M}$ et al (2010). Increased synaptic dopamine function in associative regions of the striatum in schizophrenia. Arch Gen Psychiatry 67: 231-239.

Kegeles LS, Abi-Dargham A, Zea-Ponce Y, Rodenhiser-Hill J, Mann JJ, Van Heertum RL et al (2000). Modulation of amphetamine-induced striatal dopamine release by ketamine in humans: implications for schizophrenia. Biol Psychiatry 48: 627-640.

Kegeles LS, Martinez D, Kochan LD, Hwang DR, Huang Y, Mawlawi O et al (2002). NMDA antagonist effects on striatal dopamine release: positron emission tomography studies in humans. Synapse 43: 19-29.

Krystal JH, D'Souza DC, Karper LP, Bennett A, Abi-Dargham A, Abi-Saab D et al (1999). Interactive effects of subanesthetic ketamine and haloperidol in healthy humans. Psychopharmacology 145: 193-204.

Krystal JH, Karper LP, Seibyl JP, Freeman GK, Delaney R, Bremner JD et al (1994). Subanesthetic effects of the noncompetitive NMDA antagonist, ketamine, in humans. Psychotomimetic, perceptual, cognitive, and neuroendocrine responses. Arch Gen Psychiatry 51: 199-214.

Lahti AC, Koffel B, LaPorte D, Tamminga CA (1995). Subanesthetic doses of ketamine stimulate psychosis in schizophrenia. Neuropsychopharmacology 13: 9-19.

Moghaddam B, Adams B, Verma A, Daly D (1997). Activation of glutamatergic neurotransmission by ketamine: a novel step in the pathway from NMDA receptor blockade to dopaminergic and cognitive disruptions associated with the prefrontal cortex. J Neurosci 17: 2921-2927.

Morgan CJ, Muetzelfeldt L, Curran HV (2010). Consequences of chronic ketamine self-administration upon neurocognitive function and psychological wellbeing: a 1-year longitudinal study. Addiction 105: 121-133.

Murphy K, Birn RM, Handwerker DA, Jones TB, Bandettini PA (2009). The impact of global signal regression on resting state correlations: are anti-correlated networks introduced? Neuroimage 44: 893-905.

O’Doherty J, Dayan P, Schultz J, Deichmann R, Friston K, Dolan RJ (2004). Dissociable roles of ventral and dorsal striatum in instrumental conditioning. Science 304: 452-454.

Olney JW, Farber NB (1995). Glutamate receptor dysfunction and schizophrenia. Arch Gen Psychiatry 52: 998-1007.

Pantelis C, Barnes TRE, Nelson HE (1992). Is the concept of frontal-subcortical dementia relevant to schizophrenia? $\mathrm{Br} \mathrm{J}$ Psychiatry 160: 442-460.

Parent A, Hazrati LN (1995). Functional anatomy of the basal ganglia. II. The place of subthalamic nucleus and external pallidum in basal ganglia circuitry. Brain Res Brain Res Rev 20: 128-154.

Peters ER, Joseph SA, Garety PA (1999). Measurement of delusional ideation in the normal population: Introducing the PDI (Peters et al Delusions Inventory). Schizophr Bull 25: 553-576.

Pettersson-Yeo W, Allen P, Benetti S, McGuire P, Mechelli A (2011). Dysconnectivity in schizophrenia: where are we now? Neurosci Biobehav Rev 35: 1110-1124.

Pomarol-Clotet E, Honey GD, Murray GK, Corlett PR, Absalom AR, Lee $\mathrm{M}$ et al (2006). Psychological effects of ketamine in healthy volunteers. Phenomenological study. Br J Psychiatry 189: 173-179.

Satterthwaite TD, Elliott MA, Gerraty RT, Ruparel K, Loughead J, Calkins ME et al (2013). An improved framework for confound regression and filtering for control of motion artifact in the preprocessing of resting-state functional connectivity data. Neuroimage 64: 240-256.

Scheidegger M, Walter M, Lehmann M, Metzger C, Grimm S, Boeker $\mathrm{H}$ et al (2012). Ketamine decreases resting state functional network connectivity in healthy subjects: implications for antidepressant drug action. PLoS One 7: e44799.

Smith DJ, Bouchal RL, Desanctis CA, Monroe PJ, Amedro JB, Perrotti JM et al (1987). Properties of the interaction between ketamine and opiate binding sites in vivo and in vitro. Neuropharmacology 26: 1253-1260. 
Song XW, Dong ZY, Long XY, Li SF, Zuo XN, Zhu CZ et al (2011). REST: a toolkit for resting-state functional magnetic resonance imaging data processing. PLoS One 6: e25031.

Van Dijk KRA, Hedden T, Venkataraman A, Evans KC, Lazar SW, Buckner RL (2010). Intrinsic functional connectivity as a tool for human connectomics: theory, properties, and optimization. J Neurophysiol 103: 297-321.

Van Dijk KRA, Sabuncu MR, Buckner RL (2012). The influence of head motion on intrinsic functional connectivity MRI. Neuroimage 59: 431-438.
Ventura JLD, Nuechterlein KH, Lieberman RP, Green M, Shaner A (1993). Brief Psychiatric Ratings Scale (BPRS) expanded version (4.0) scales, anchor points and administration manual. Int $J$ Methods Psychiatr Res 3: 227-243.

Vollenweider FX, Leenders KL, Scharfetter C, Antonini A, Maguire P, Missimer J et al (1997). Metabolic hyperfrontality and psychopathology in the ketamine model of psychosis using positron emission tomography (PET) and [18F]fluorodeoxyglucose (FDG). Eur Neuropsychopharmacol 7: 9-24.

Supplementary Information accompanies the paper on the Neuropsychopharmacology website (http://www.nature.com/npp) 